\title{
STABILITY OF THE BISHOP FAMILY OF HOLOMORPHIC DISCS
}

\author{
WILHELM KLINGENBERG \\ Mathematisches Institut, Universität Tübingen \\ Auf der Morgenstelle 10, 72076 Tübingen, Germany \\ E-mail: wilhelm@moebius.mathematik.uni-tuebingen.de
}

1. A point $p$ on an embedded $C^{\infty}$-smooth real surface $M \hookrightarrow \mathbb{C}^{2}$ is said to have a complex tangent if $T_{p} M$ is a complex line in $T_{p} \mathbb{C}^{2}$. If this is the case one can choose complex coordinates $z, w=u+i v$ on $\mathbb{C}^{2}$ such that $T_{p} M$ is the $z$-axis and $p=(0,0)$. Then $M$ is locally a graph

$$
w=f(z)
$$

where $f$ is a smooth complex valued function that vanishes of second order at the origin. If $\partial^{2} f / \partial z \partial \bar{z}(0) \neq 0$, then a quadratic holomorphic substitution of $z, w$ normalizes the second-order part of $f$ at the origin to

$$
q(z)=\gamma\left(z^{2}+\bar{z}^{2}\right)+z \bar{z}
$$

where $\gamma \geq 0$ is Bishop's invariant [B]. Then we have

$$
f=q+h+i k
$$

where $h, k$ are real functions that vanish of third order at 0 . Let $\pi: \mathbb{C}^{2} \rightarrow\{v=0\} \subseteq \mathbb{C}^{2}$, $\pi(z, w)=(z, u), M_{0}=\pi M$, and assume that $q: \mathbb{C} \rightarrow \mathbb{R}$ is positive definite, i.e.

$$
0 \leq \gamma<1 / 2 \text {. }
$$

In this case $0 \in M$ is called an elliptic point. By rescaling $z, w$ we may assume that

$$
\begin{gathered}
h, k \in C^{\infty}(\bar{\Delta} ; \mathbb{R}), h, k=O\left(|z|^{3}\right), \\
q+h \quad \text { is convex on } \bar{\Delta}, \\
1 \leq(q+h)(z) \text { on } \partial \bar{\Delta} .
\end{gathered}
$$

Now $M_{0}$ bounds the hypersurface $D_{0}=\{(q+h)(z)<u, v=0\}$ which in turn is foliated by the holomorphic $\operatorname{discs}\{(q+h)(z)<t, w=t, 0<t<1\}$ whose boundaries lie in $M_{0}$.

1991 Mathematics Subject Classification: Primary: 58D27. Secondary: 58G20.

The paper is in final form and no version of it will be published elsewhere. 
This family of holomorphic discs survives in a possibly smaller neighbourhood of $0 \in M$ by Bishop's Theorem [B].

In this note we show that this foliated hypersurface depends smoothly on $M$ in $C^{\infty}$ Fréchet topology and closely follow $[\mathrm{KW}]$.

Stability of holomorphic discs with boundary in totally real surfaces has been studied in $[\mathrm{Be}],[\mathrm{F}],[\mathrm{K}],[\mathrm{O}]$. It is relevant in constructions for fillable contact structures on $S^{3}$, see $[\mathrm{EK}],[\mathrm{H}]$. The present paper is organized as follows. In the second section we state the stability result. In the third we give smooth dependence of the Hilbert transform on the boundary in the form that it enters the present problem. In the last section we show how the result follows from the estimate of Section 3 and a normalization procedure of the real surface.

2. For $l \in \mathbb{N}, l \geq 3$ let $\mathfrak{M}_{l}=\{w=(q+h+i k)(z) ; q, h, k$ satisfying $(1)-(5)$ and $k=$ $\left.O\left(|z|^{l}\right)\right\}$ and let $\mathfrak{M}_{0}=\{w=(q+h)(z) ; q, h$ satisfying $(1)-(5)\}$ both equipped with the $C^{\infty}(\bar{\Delta})$ Fréchet topology. We have $\pi: \mathfrak{M}_{l} \rightarrow \mathfrak{M}_{0}, \pi M=M_{0}, M_{0}^{\epsilon}=M_{0} \cap\{u<\epsilon\}$, and $M_{0}^{\epsilon}$ bounds $D_{0}^{\epsilon}=\{(q+h)(z)<u<\epsilon, v=0\}$. Here and later $M \in \mathfrak{M}_{l}$ is identified with its graph function $f=q+h+i k$. The main result of [KW] now reads as follows.

Theorem $1[\mathrm{KW}]$. Let $M \in \mathfrak{M}_{3}$ then there exists $\epsilon>0$ and a $C^{\infty}$ embedding $G$ : $\left(D_{0}^{\epsilon}, M_{0}^{\epsilon}\right) \rightarrow\left(\mathbb{C}^{2}, M\right)$ such that
a) $G(0)=0, d G(0)=\left[\begin{array}{c}\mathrm{Id} \\ 0\end{array}\right]$ where Id $:\{v=0\} \rightarrow\{v=0\}$ is the identity.
b) $u \circ G(0, u)=u$
c) $G \mid w=r^{2}$ is holomorphic for all $r^{2}<\epsilon$.

To formulate the smooth dependence of $G$ from $M$ we introduce more notation. Let $\mathfrak{G} \rightarrow \mathfrak{M}_{3}$ be the locally trivial fibre bundle over $\mathfrak{M}_{3}$ whose fibre over $M \in \mathfrak{M}$ is given by $\mathfrak{G}_{M}=\left\{G:\left(D_{0}^{\epsilon}, M_{0}^{\epsilon}\right) \hookrightarrow\left(\mathbb{C}^{2}, M\right) ; C^{\infty}\right.$-smooth, some $\left.\epsilon>0\right\}$ and equip it with the $C^{\infty}$ Fréchet topology.

TheOREM 2. There exists a continuous function $\epsilon: \mathfrak{M}_{3} \rightarrow \mathbb{R}^{+}$and a $C^{\infty}$-smooth section $p$ of $\mathfrak{G}$ whose values satisfy $\mathrm{a}), \mathrm{b}), \mathrm{c})$ from Theorem 1.

3. Let $q+h \in \mathfrak{M}_{0}$, then denote by $\Delta_{q+h}=\{z \in \Delta:(q+h)(z)<1\}$. We associate to $\Delta_{q+h}$ the diffeomorphism $g(q+h): \Delta \rightarrow \Delta_{q+h}$ that maps $\left\{|z|^{2}=t\right\}$ to $\{(q+h)(z)=t\}$ for $0 \leq t \leq 1$ and preserves the rays through 0 . The existence and smoothness of $g$ in $z$ and smooth dependence on $q+h$ follows from Morse Lemma and (5). We have $g: \mathfrak{M}_{0} \times(0,1) \rightarrow C^{\infty}\left(S^{1}, \mathbb{C}\right)$ defined by $g(q+h, r)=g(q+h)(\cdot, r)$, where we introduced polar $(0, r)$ coordinates on $\Delta$, and $g_{0}(q+h, r):=g(q+h, r)-g(q, r)$. In this regard one has the following

Proposition 3. The map $g: \mathfrak{M}_{0} \times(0,1) \rightarrow C^{\infty}\left(S^{1} ; \mathbb{C}\right)$ is smooth and there exists $K=K(q+h, j, s)>0$ which is locally bounded in $q+h$ such that

a) $|g|_{j},\left|g_{0} / r\right|_{j} \leq K \cdot r$

b) $\left|\partial_{r}^{s} g\right|_{j}, \mid \partial_{r}^{s}\left(g_{0} /\left.r\right|_{j} \leq K\right.$.

Here $g=g(q+h, r) \in C^{\infty}\left(S^{1} ; \mathbb{C}\right)$ and $|\cdot|_{j}$ denotes the $C^{j}$-sup norm. 
For $(q+h, r) \in \mathfrak{M}_{0} \times(0,1),(j, \alpha) \in \mathbb{N} \times(0,1)$ let $H(q+h, r) \in \operatorname{Hom} C^{j, \alpha}\left(S^{1}, \mathbb{R}\right)$ be the Hilbert transform operator on the closed convex curve $\left\{(q+h)(z)=r^{2}\right\} \subseteq \Delta$, parametrized by $\theta \in S^{1}$. It maps $\xi \in C^{j, \alpha}\left(S^{1}, \mathbb{R}\right)$ to its harmonic conjugate with mean zero. The main technical point in the proof of Theorem 1 and 2 lies in the analysis of the asymptotic behaviour of $H$ on the curves $\left\{(q+h)(z)=r^{2}\right\}$ as $r^{2} \rightarrow 0$. The functions $g$, $g_{0}$ enter in the integral operator representations of $H$, see $(2.7)$ of $[\mathrm{KW}]$.

Proposition 4. The map $H: \mathfrak{M}_{0} \times(0,1) \rightarrow \operatorname{Hom} C^{j, \alpha}\left(S^{1}, \mathbb{R}\right)$ is continuous and there exists $K=K(q+h, j, s)>0$, locally bounded in the first argument, such that

a) $|H(q+h, r)-H(q, r)|_{j, \alpha} \leq K \cdot r$

b) $\left|\partial_{r}^{s} H(q+h, r)\right|_{j, \alpha} \leq K$.

Given Proposition 3, the above propositions follow immediately from Lemma 2.2 and Theorem 2.5 of $[\mathrm{KW}]$.

4. For the proof of Theorem 2 one represents $G=p(M) \in \mathfrak{G}_{M}$ by the ansatz

$$
G(z, w)=(z+z A(z, r), w+B(z, r))
$$

where $u=r^{2}, A, B$ are holomorphic in $z$ on $\left\{(q+h)(z)<r^{2}\right\} \subset \Delta, \operatorname{Re} B(0, r)=0$, $\operatorname{Im} A(0, r)=0$, and one stipulates that $G$ maps $\left\{(q+h)(z)=r^{2}, u=r^{2}\right\} \subset M_{0}$ into $M=\{w=(q+h+i k)(z)\}$, which may be written as

$$
r^{2}+B(z, r)=(q+h+i k)(z+z A(z, r))
$$

for $(q+h)(z)=r^{2}$. This functional equation needs to be solved for $A, B$, these extended holomorphically to the interior of $(q+h)(z)=r^{2}$, and shown to depend smoothly on $q+h+i k \in \mathfrak{M}_{3}$. Using Hilbert transform one may eliminate $B$ from (7) and is lead to the following:

$$
F(q+h+i k, r, \xi)=0
$$

where $F: \mathfrak{M}_{3} \times(0,1) \times C^{j, \alpha}\left(S^{1}\right) \rightarrow C^{j, \alpha}\left(S^{1}\right)$ is defined by

$$
F=(q+h)(z+z A(q+h, r)[\xi])-r^{2}+H(q+h, r)[k(z+z A(q+h, r)[\xi]] .
$$

Here,

$$
A(q+h, r)[\xi]=\xi+i H(q+h, r)[\xi]
$$

and $(q+h)(z)=r^{2}$. By Propositions 3 and $4, F$ is smooth. As in [KW, $\left.\S 1\right]$ it follows that if $\xi_{r} \in C^{j, \alpha}\left(S^{1}\right), r \in(0,1)$ satisfies $(8)$, then

$$
\begin{aligned}
& A(z, r)=A(q+h, r)[\xi] \\
& B(z, r)=-r^{2}+(q+h+i k)(z+z A(z, r))
\end{aligned}
$$

satisfy (7) and its holomorphic extensions in (6) will give a point $G \in \mathfrak{G}_{M}$ satisfying a), b), c) of Theorem 1. Applying a Picard iteration scheme to solve (8) for $\xi_{r}$ leads to the following existence result $[\mathrm{KW}, 3.3]$ :

Proposition 5. Let $(j, \alpha) \in \mathbb{N} \times(0,1), l \geq 3$. Then there exists a continuous function $\epsilon: \mathfrak{M}_{l} \rightarrow \mathbb{R}^{+}$and a smooth map $\left\{M \times(0, \epsilon(M)) ; M \in \mathfrak{M}_{3}\right\} \rightarrow C^{j, \alpha}\left(S^{1}\right): q+h+i k \rightarrow \xi_{r}$, $r^{2}>\epsilon^{2}(M)$ with

a) $F(q+h+i k, r, \xi r)=0$ 
b) $\left|\partial_{r}^{s} \xi r\right|_{j} \leq K r^{l-2-s}$

where $K=K(l, j, s, q+h+i k)>0$ is locally bounded in $\mathfrak{M}_{3}$.

Now by extending $\xi_{r}$ to $\left\{(q+h)(z)<r^{2}\right\}$ harmonically and by (6), (10) one concludes the following as in Theorem 4.2, [KW].

THEOREM 6. Let $l \geq 7, s=(l-7) / 3$, then there exists a continuous section $p(l)$ of $\mathfrak{G}^{s} \rightarrow \mathfrak{M}_{l}$ satisfying $\left.\left.\left.\mathrm{a}\right), \mathrm{b}\right), \mathrm{c}\right)$ of Theorem 1 , where $\mathfrak{G}^{s}$ is as in the second section but with $C^{s}$-regularity instead of $C^{\infty}$-regularity.

Now Theorem 2 follows from Theorem 6 , boundary regularity of holomorphic discs in totally real surfaces, and the following

Proposition 7. Let $\operatorname{Hol}\left(\mathbb{C}^{2}, 0\right)$ be the manifold of germs at 0 of holomorphic invertible maps of $\left(\mathbb{C}^{2}, 0\right)$ fixing the origin, endowed with Fréchet topology. Then for any $l \geq 3$ there exists a continuous map $h: \mathfrak{M}_{3} \rightarrow \operatorname{Hol}\left(\mathbb{C}^{2}, 0\right), M \rightarrow h_{M}$, such that $h_{M}(M) \in \mathfrak{M}_{l}$.

\section{References}

[Be] E. Bedford, Levi flat hypersurfaces in $\mathbb{C}^{2}$ with prescribed boundary: Stability, Ann. Scuola Norm. Sup. Pisa Cl. Sci. (4) 9 (1981), 529-570.

[B] E. Bishop, Differentiable manifolds in complex Euclidean space, Duke Math. J. 32 (1965), 1-22.

[EK] Y. Eliashberg, W. Klingenberg, Fillable contact structures on $S^{3}$, in preparation.

[F] F. Forstneric, Analytic discs with boundaries in a maximal real submanifold of $\mathbb{C}^{2}$, Ann. Inst. Fourier (Grenoble) 37 (1987), 1-44.

[H] H. Hofer, Pseudoholomorphic curves in symplectizations with applications to the Weinstein conjecture in dimension three, Invent. Math. 114 (1993), 515-563.

[KW] C. Kenig, S. Webster, The local hull of holomorphy of a surface in the space of two complex variables, Invent. Math. 67 (1982), 1-21.

[K] W. Klingenberg, Moduli space of holomorphic discs with boundary, to appear in Arch. Math. (Basel).

[O] Y.-G. Oh, Riemann-Hilbert problem and application to the perturbation theory of analytic discs, to appear in Math. Z. 